\title{
Prediction and production of simple mathematical equations \\ ${ }^{1}$ Max Planck Institute for Psycholinguistics, Nijmegen, The Netherlands \\ ${ }^{2}$ International Max Planck Research School for Language Sciences, Nijmegen, The Netherlands \\ ${ }^{3}$ Donders Institute for Brain, Cognition, and Behavior, Radboud University, Nijmegen, The Netherlands
}

\section{Introduction}

- An important issue in current psycholinguistics is the relationship between the language production and the language comprehension system.

- While traditional theories [e.g., 1] assume two separate systems, a recent framework [2] proposed an integration of both: Speakers construct action plans to predict their own utterances; listeners internally simulate the other person's speech using their production system and predict upcoming content at various levels.

- In both modalities the language production system constructs forward models, which are compared to the actual language output.

\section{The current study}

- Using simple mathematical equations, we compared language production and language prediction processes and tested the suggested involvement of the production system in language comprehension.

- Mathematical equations are similar to proper sentences but allow for tight control of lexical and grammatical variables (e.g., word frequency, plausibility, constituent dependencies, etc.).

\section{Hypotheses}

- According to traditional theories, production and comprehension draw upon separate systems and should evoke similar but not identical behavior. If the production system is involved in production and comprehension, there should be no difference between these processes.

\section{Method}
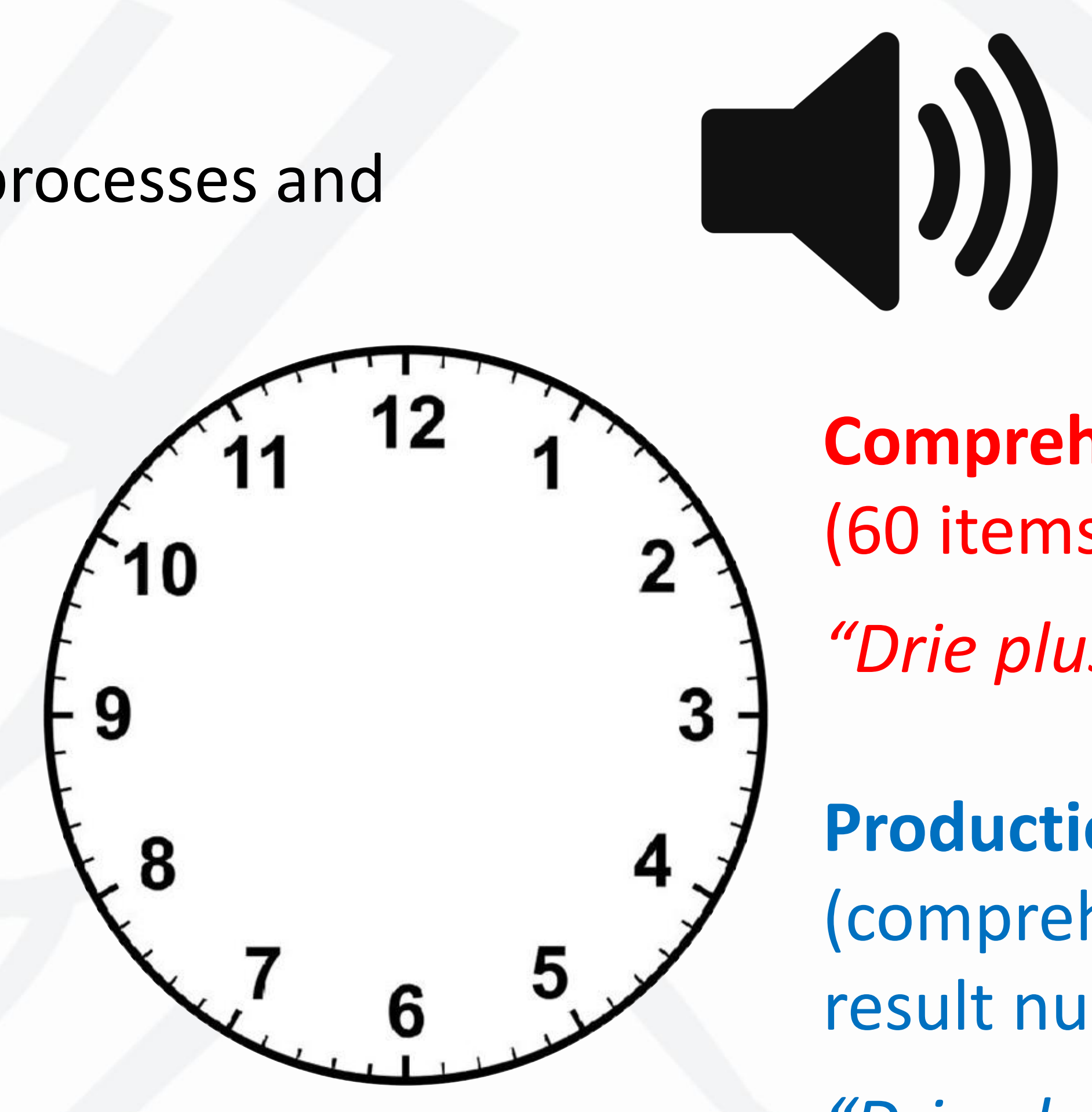

- Participants heard mathematical equations and looked at a clock face showing the numbers 1 to 12.

- On alternating trials they either heard a complete equation or they heard the first part and had to produce the solution themselves.

- Participants were encouraged to look at the relevant numbers throughout the trial.

\section{Experiment 1}

- 24 native Dutch university students carried out the experiment in their mother tongue.

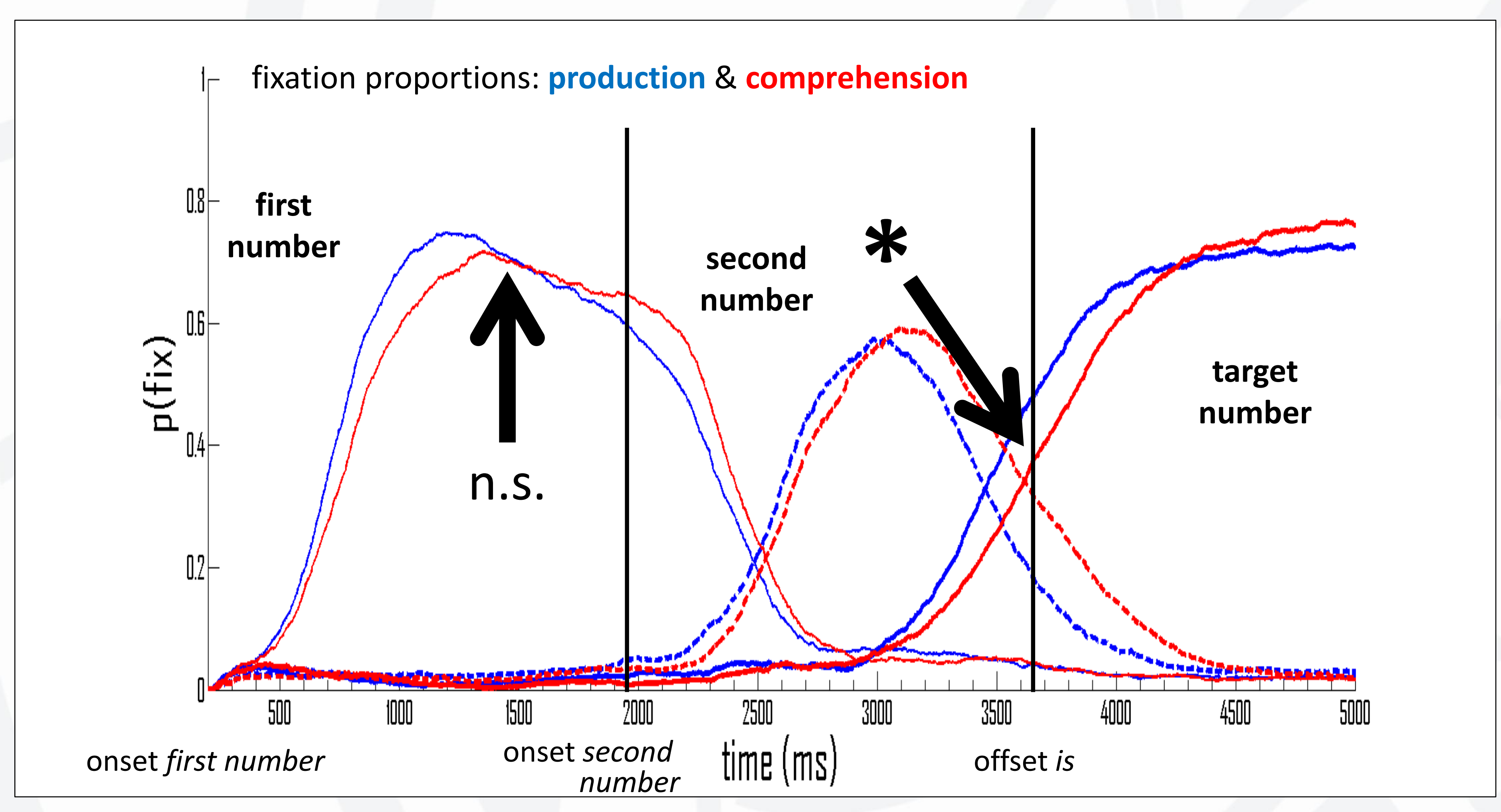

During the predictive window (onset second number - offset is):

Comprehension: participants looked at the target number

before it was mentioned by the speaker.

Production: participants looked at the target number before they named it.

- Participants looked earlier at the target when they had to speak themselves than when they listened.

\section{Conclusion}

- Predicting during listening and planning to speak are indeed very similar but not identical.

- Attentional shifts to targets happened earlier when speech was planned compared to when speech was predicted.

\section{Open issues}

Do these differences scale up to more complex linguistic utterances? Is target number production/prediction comparable to proper language processing?

\section{Experiment 2}

- 24 bilingual German-Dutch university students carried out the experiment in Dutch (L2).

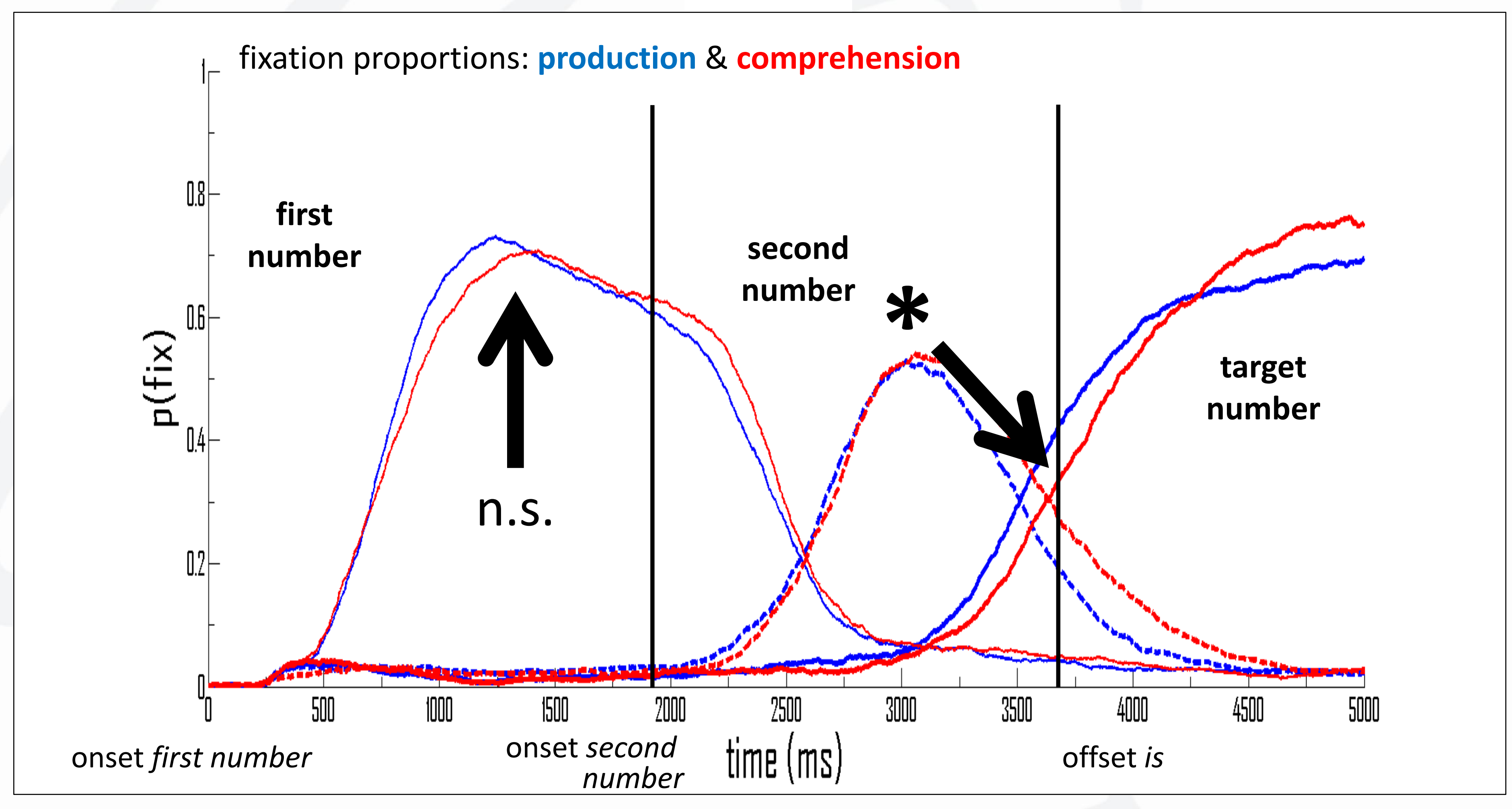

- Speech onset latencies for target numbers were longer when being produced by the participants as compared to the recorded speaker

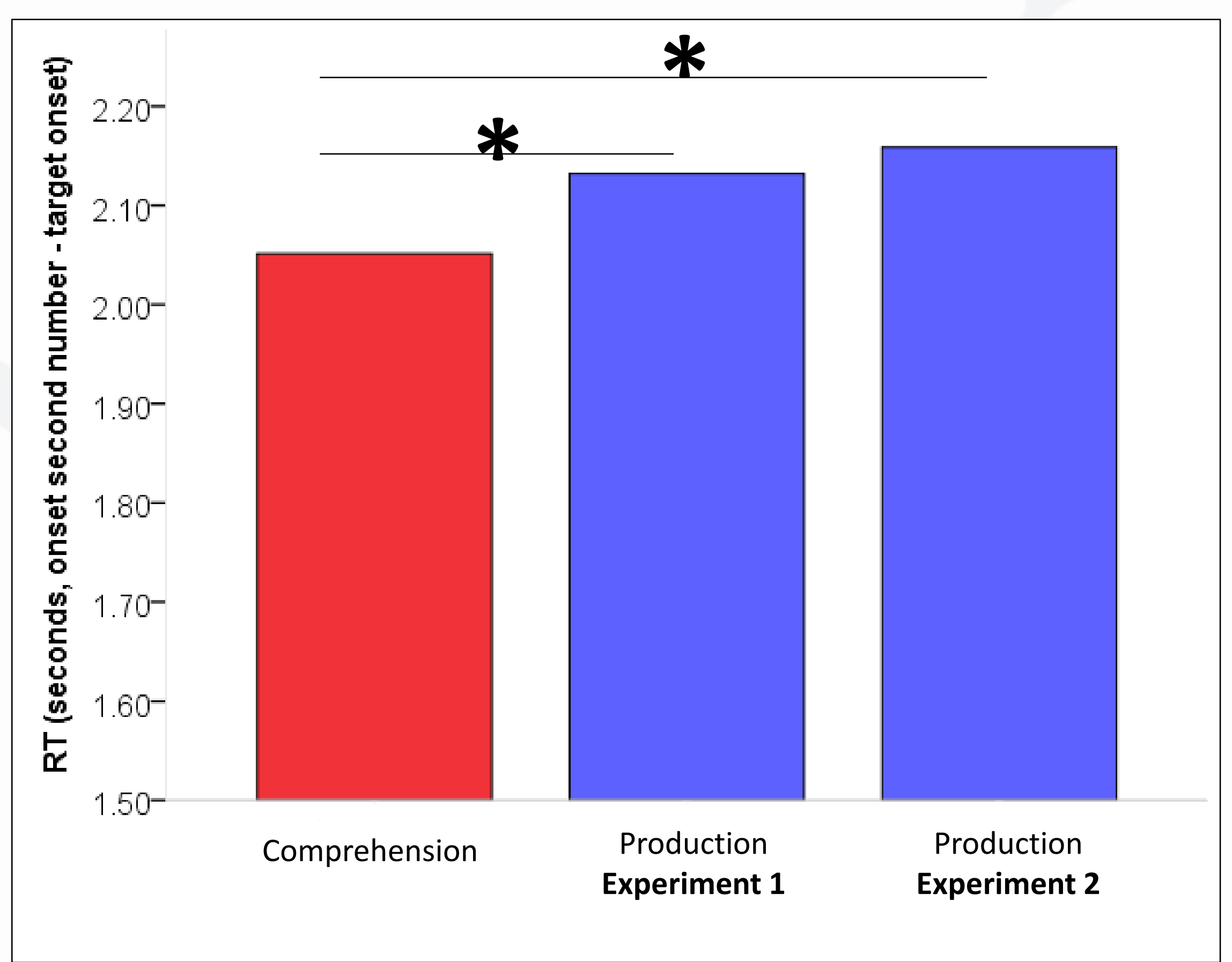

\section{References}

[1] Clark, H. H., \& Clark, E. V. (1977). Psychology and language: An introduction to psycholinguistics. New York: Harcourt Brace Jovanovich.

[2] Pickering, M. J., \& Garrod, S. (2013). An integrated theory of language production and comprehension. Behavioral and Brain Sciences, FirstView, 1-19. doi:10.1017/S0140525X12001495 